\title{
Can exercise rehabilitation evolve into a new therapeutic area?
}

\author{
Chang-Ju Kim (iD http://orcid.org/0000-0003-4749-5795
}

Traditionally, exercise rehabilitation has been shown to be beneficial for the muscle and bone diseases. In present issue of the Journal of Exercise Rehabilitation (JER), the effects of exercise on bone deformity, muscle strength, and pulmonary hypertension, etc., are announced. Interestingly, some articles concerning the efficacies of exercise on neuropsychiatric disorders are published in this issue.

Exercise is known to possess antiapoptotic, antidepressive, and neurogenesis effects, and the possibility that exercise rehabilitation might be extended to the therapeutic area for neuropsychiatric disorders has been suggested. Especially, depression has emerged as a new challenging disease for modern society. Although many types of antidepressants have been developed, depression is still one of the mental illnesses that are difficult to be cured, and the medical-economic complications of depression lead to very large social problems, in addition to personal problems. Verifying the antidepressant effect of exercise would be an opportunity to open up new horizon of exercise rehabilitation. Clinical trials are needed to validate the efficacy of exercise on depressive patients as well as animal studies for the evaluation of the mechanisms of exercise on depression.
In this issue, it has been reported that exercise rehabilitation is effective for depression caused by various etiologies. The valuable articles recruited in this issue show the possibility of the exercise rehabilitation to evolve as the new therapeutic technology for depressive disorders.

JER aims to expand and deliver knowledge of exercise and to explore the possibility of exercise to move into new therapeutic area. The Editorial Board of JER would like to acknowledge gratitude to the authors for the valuable articles submitted to this issue of the JER.

\section{CONFLICT OF INTEREST}

No potential conflict of interest relevant to this article was reported.

President of the Korean Society of Exercise Rehabilitation Department of Physiology, College of Medicine, Kyung Hee University, 26 Kyungheedae-ro, Dongdaemun-gu, Seoul 02447, Korea E-mail: changju@khu.ac.kr 